NATIONAL LABORATORY

MANAGED BY UT-BATTELLE

FOR THE DEPARTMENT OF ENERGY

\title{
THE COVARIANCE AND BICOVARIANCE OF THE STOCHASTIC NEUTRON FIELD
}

\author{
R. B. Perez \\ J. K. Mattingly \\ T. E. Valentine \\ J. T. Mihalczo
}

October 2000

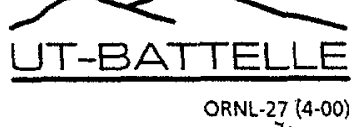


ORNL/TM-13690

\title{
THE COVARIANCE AND BICOVARIANCE OF THE STOCHASTIC NEUTRON FIELD
}

\author{
R. B. Perez \\ J. K. Mattingly \\ T. E. Valentine \\ J. T. Mihalczo \\ Oak Ridge National Laboratory* \\ P.O. Box 2008 \\ Oak Ridge, Tennessee 37831-6004, U.S.A. \\ (423) 574-5571
}

October 2000

- Managed by UT-Battelle, LLC for the U.S. Department of Energy under contract DE-AC05-00OR22725. 
This page intentionally left blank. 


\section{SYMBOLS AND NOTATION}

$\vec{r}_{q}=$ qth detector location

$\mathrm{v}=$ neutron speed

$V_{q o}=$ detector volume

$\Delta_{q i}=$ gate length during which the qth detector is on $(\sim \mathrm{sec})$

$t_{\text {qf }}=$ final time when the qth detector is turned off

$\gamma_{q}=$ qth detector efficiency (counts/event)

$K_{q}(t)=$ qth detector impulse response function $\left(\mathrm{sec}^{-1}\right)$

$A_{q}=$ short hand notation for the set of symbols $\left(\Delta_{\mathrm{qc}}, \mathrm{t}_{\mathrm{qf}}\right)$

$A_{2}=$ short hand notation for the set $\left(\mathrm{A}_{1}, \mathrm{~A}_{2}\right)$

$A_{3}=$ short hand notation for the set $\left(\mathrm{A}_{1}, \mathrm{~A}_{2}, \mathrm{~A}_{3}\right)$

$L_{o}, L_{o}^{+}=$forward and adjoint transport operators respectively

$\vec{n}=$ unit normal vector

$F_{c}=$ average spontaneous fission rate of the neutron source $\left(\frac{\text { source fission }}{\mathrm{sec}}\right)$

$\chi_{s}(\mathrm{v})=$ normalized source fission neutron spectrum

$\chi(\mathrm{v})=$ normalized fissile material fission neutron spectrum

$\Sigma_{T}=$ total neutron cross section

$\Sigma_{s j}, \Sigma_{c}, \Sigma_{F}=$ neutron scattering, capture and fission cross sections

$\Sigma_{o q c}=$ neutron capture cross section for the qth detector

$f\left(v^{\prime} \vec{\Omega}^{\prime} \mid v \vec{\Omega}\right)=$ scattering probability function (normalized, dimensionless)

$\Sigma_{s}\left(\mathrm{v}^{\prime} \Omega^{\prime} \mid \mathrm{v} \Omega\right)=$ scattering kernel

$C_{o}(\vec{r}, \mathrm{v}, \mathrm{t})=$ probability for the occurrence of a capture event $=\Sigma_{c} / \Sigma_{T}$

$C_{q}(\vec{r}, \mathrm{v}, \mathrm{t})=$ probability for the occurrence of a capture event within the qth detector volume

$\varepsilon_{s j}=$ probability of emission of $\mathrm{j}$-neutrons per source fission event

$\varepsilon_{j}=$ probability of emission of $j$-neutrons per fission event in the fuel

$C_{j}=$ probability that a neutron interaction with the fuel material generates $\mathrm{j}$-prompt

$$
\text { neutrons }=\varepsilon_{j} \Sigma_{F} / \Sigma_{T}
$$

$\mathrm{d} \underline{\mathrm{r}}=$ space volume element

$\mathrm{dv} d \Omega=$ speed and angular volume element

$<\mathrm{f}|\mathrm{g}\rangle=$ time-phase space inner product

$$
=\int_{-\infty}^{\infty} \mathrm{dt} \int \mathrm{d} \underline{\mathrm{r}} \mathrm{dv} \mathrm{d} \Omega \mathrm{f}(\overrightarrow{\mathrm{r}}, \mathrm{v}, \vec{\Omega}, \mathrm{t}) \mathrm{g}(\overrightarrow{\mathrm{r}}, \mathrm{v}, \vec{\Omega}, \mathrm{t})
$$

$(\mathrm{f} \mid \mathrm{g})=$ phase-space inner product $=\int \mathrm{d} \underline{\mathrm{r}} \mathrm{dv} d \Omega \mathrm{f}(\overrightarrow{\mathrm{r}}, \mathrm{v}, \vec{\Omega}, \mathrm{t}) \mathrm{g}(\overrightarrow{\mathrm{r}}, \mathrm{v}, \vec{\Omega}, \mathrm{t})$ 
This page intentionally left blank. 


\section{CONTENTS}

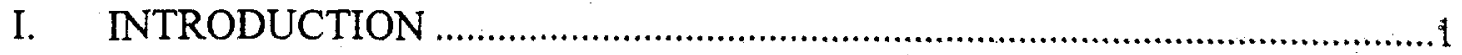

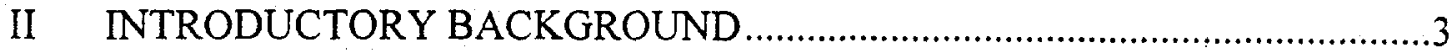

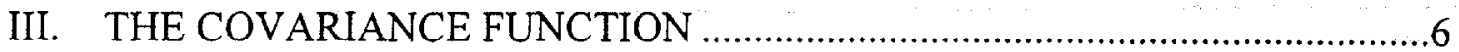

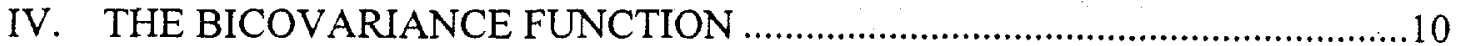

V. THE TWO AND THREE DETECTORS CROSS CORRELATION FUNCTIONS

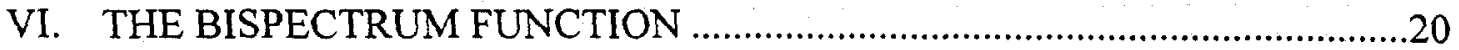

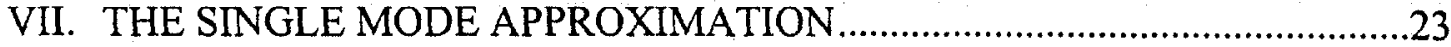

VIII. SUMMARY AND DISCUSSION

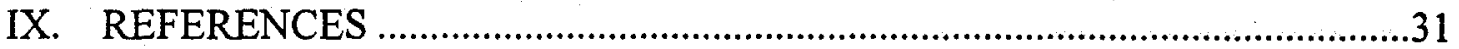

APPENDIX A

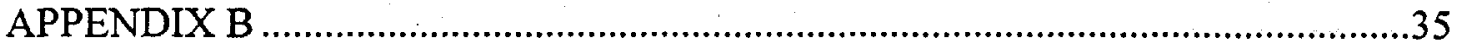

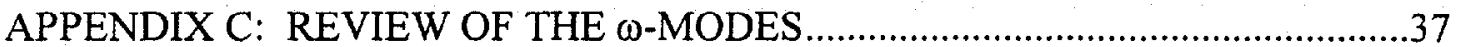


This page intentionally left blank. 


\section{INTRODUCTION}

The use of stochastic neutron field theory (neutron noise) for the measurement of reactor physics parameters goes back to the early work of Serber ${ }^{1}$, Feynmann $^{2}$, and Orndoff ${ }^{3}$. Since then, a large variety of methods and applications has been developed. In the majority of these methods, some form of modified one-point reactor kinetics was used for the interpretation of the measurements. In fact, the high level of sophistication of the instrumentation used was not matched by the theory.

In 1965, Bell ${ }^{4}$ developed a general theory of the stochastic neutron field, and in 1987, Munoz-Cobo et $\mathrm{al}^{5}$ enlarged this treatment to include the effect of the detectors in the neutron field. In both instances, the complexity of the theoretical results were beyond the computing capabilities then available thus, the mismatch between experimental and theoretical methods remained in existence because the powerful Monte-Carlo methods then at work, were only applicable to static neutron fields. This problem was eliminated by the development of a time-dependent Monte-Carlo code specially written by T. E. Valentine ${ }^{6}$ for the analysis of stochastic measurements that gave them relevance to the results of the general theory. The purpose of this work is to illustrate the derivation of observables of the stochastic neutron filed from its general treatment. ${ }^{5}$

We start with some preliminary developments by introducing the $\mathrm{L}_{\mathrm{o}}$-operator and its adjoint $L_{0}^{+}$.

$$
L_{o}=L_{o o}-F_{F} ; L_{o}^{+}=L_{o o}^{+}-F_{F}^{+}
$$

with 


$$
\begin{aligned}
& L_{o o}=\vec{\Omega} \cdot \vec{\nabla}+\sum_{T}-\int \mathrm{d} v^{\prime} \mathrm{d} \Omega^{\prime} \sum_{s}\left(\mathrm{v}^{\prime} \vec{\Omega}^{\prime} / \mathrm{v} \vec{\Omega}\right) \\
& F_{F}=\bar{v} \frac{\chi(\mathrm{v})}{4 \pi} \int \mathrm{dv}^{\prime} \mathrm{d} \Omega^{\prime} \Sigma_{\mathrm{F}}\left(\mathrm{v}^{\prime}\right) \\
& L_{o o}^{+}=-\vec{\Omega} \cdot \vec{\nabla}+\sum_{T}-\int \mathrm{d} \mathrm{v}^{\prime} \mathrm{d} \Omega^{\prime} \sum_{s}^{+}\left(\mathrm{v}^{\prime} \vec{\Omega} \mid \mathrm{v} \vec{\Omega}\right) ; \sum_{s}^{+}\left(\mathrm{v}^{\prime} \vec{\Omega} \mid \mathrm{v} \vec{\Omega}\right)=\Sigma_{s}\left(\mathrm{v} \vec{\Omega} \mid \mathrm{v}^{\prime} \vec{\Omega}\right)(1.4) \\
& F_{F}^{+}=\bar{v}_{p} \Sigma_{F}(\mathrm{v}) \int \mathrm{dv^{ \prime }} \mathrm{d} \Omega^{\prime} \frac{\chi\left(\mathrm{v}^{\prime}\right)}{4 \pi}
\end{aligned}
$$

We also use the operators

$$
T_{R}=\int \mathrm{dv} \mathrm{d} \Omega \frac{\chi(\mathrm{v})}{4 \pi} ; T_{s}=\int \mathrm{dv} \mathrm{d} \Omega \frac{\chi_{s}(\mathrm{v})}{4 \pi}
$$

and

$$
O_{s}=\frac{1}{\Sigma T} \int \mathrm{dv}^{\prime} \mathrm{d} \Omega^{\prime} \Sigma_{s}^{+}
$$

The commutation relation between the forward operator $\left\{\frac{1}{\mathrm{v}} \frac{\partial}{\partial t}+L_{o}\right\}$ and its adjoint

$$
\begin{aligned}
& \left\{-\frac{1}{\mathrm{v}} \frac{\partial}{\partial t}+L_{o}^{+}\right\} \text {is given below: } \\
& \quad<\Gamma^{+}\left|\left(\frac{1}{\mathrm{v}} \frac{\partial}{\partial t}+L_{o}\right) \Gamma>=<\Gamma\right|\left(-\frac{1}{\mathrm{v}} \frac{\partial}{\partial t}+L_{o}^{+}\right) \Gamma^{+}>
\end{aligned}
$$

where, $\Gamma$, and $\Gamma^{+}$, are generalized functions that satisfy the governing equations

$$
\begin{aligned}
& \left\{\frac{1}{\mathrm{v}} \frac{\partial}{\partial t}+L_{o}\right\} \Gamma=Q_{\gamma} \\
& \left\{-\frac{1}{\mathrm{v}} \frac{\partial}{\partial t}+L_{o}^{+}\right\} \Gamma^{+}=Q_{\gamma}^{+}
\end{aligned}
$$


and where, $Q_{\gamma}, Q_{\gamma}^{+}$, are external driving functions with the proviso that the above generalized functions are such that the bilinear concomitant anociated with the commutation relation (1.8) does vanish, one obtains the useful relation.

$$
\left\langle\Gamma^{+} \mid Q\right\rangle=\left\langle\Gamma \mid Q^{+}\right\rangle
$$

Section two of this paper provides a summary of the needed results from the work of Munoz-Cobo et al. The covariance and tri-covariance functions are derived in sections three and four, respectively. Expressions for the two and three detector cross correlation functions are derived in section five. Because there is an extensive bibliography on the two-detector cross power spectral density ${ }^{7,8,9,10,11,12}$ and its derived quantity the R-ratio, this paper skips its treatment to emphasize in section six the new results, concerning the structure of the Fourier transformed three-detectors cross correlation function, that is, the bispectrum function of the source-driven neutron field. Section seven describes the single mode approximation to the bispectrum function, and section eight gives a summary . of the results obtained.

\section{INTRODUCTORY BACKGROUND}

The necessary theoretical background is provided by the work of Munoz-Cobo et $\mathrm{al}^{5}$. Here we summarize some of their results. Given, $Q_{0}$, detectors and the probability, 
$\mathrm{P}_{[\mathbb{N}\}}(\{A\})$ of finding the distribution $\{\mathrm{N}\}$ of detector counts, one defines the source probability generating function, $G_{s}$, as

$$
G_{s}(\{Z\} \mid\{A\})=\sum_{\{N\}} Z_{1}^{N} \cdots Z_{Q o}^{N_{Q \circ}} \underset{\{N\}}{P}(\{A\})
$$

The moments of, $\underset{\{N\}}{P}$, are found from the relations

$$
\left\langle N_{1} \cdots N_{q}\right\rangle=\left(\frac{\partial^{q} G_{s}}{\partial Z, \cdots \partial Z q}\right)_{1}
$$

The source probability generating function is in turn given in terms of the kernel probability generating function, $G_{K}\left(Z_{1} \cdots Z_{q} \mid \vec{X}_{1} t\right)$ :

$$
G_{s}=\exp \left\{E_{s}\right\}
$$

with

$$
\begin{aligned}
& E_{s}=\sum_{j=0}^{1_{s}} \varepsilon_{s j} Q\left[\left(F_{s}\right)^{j}-1\right] \\
& F_{s}=T_{s} G_{K}
\end{aligned}
$$

and where we introduced the operator 


$$
Q=\int_{-\infty}^{\infty} d t \int d \underline{r} S_{1}(\vec{r})
$$

and the point source

$$
S_{1}(\vec{r})=F_{c} \delta\left(\vec{r}-\vec{r}_{s}\right) \quad\left(\frac{\text { fissions }}{\mathrm{cm}^{3} \mathrm{sec}}\right)
$$

The kernel probability generating function, $G_{K}$, obeys the equation

$$
\begin{gathered}
H_{o} G_{K}(\{Z\}, \vec{x}, t)=-\Sigma_{T}\left\{C_{o}+\sum_{q=1}^{Q o} Z_{q} C_{q}+O_{s} G_{K}+\sum_{j=0}^{I} C_{j}\left(F_{R}\right)^{j}\right\} \\
H_{o}=\frac{1}{\mathrm{v}} \frac{\partial}{\partial t}+\vec{\Omega} \cdot \vec{\nabla}-\Sigma_{T} \\
F_{R}=T_{R} G_{K}
\end{gathered}
$$

and where the capture detectors are described by the probability, $\mathrm{C}_{\mathrm{q}}$, given by

$$
C_{q}\left(\vec{r}, \mathrm{v}, \mathrm{t} \mid \mathrm{A}_{q}\right)=\left(\gamma_{q} / \Sigma_{T}\right) \int_{-\infty}^{\infty} \mathrm{dt}_{1} K_{q}\left(t-t_{1}\right) U_{q}\left(t_{1} \mid t_{q f}, \Delta q c\right) \Sigma_{q c}(\mathrm{v})
$$

where the window function, $U_{q}$, is given in terms of the step function, $U_{0}$, by 


$$
U_{q}\left(t_{1} \mid A_{c}\right)=U_{o}\left(t_{1}-\left(t_{q f}-\Delta_{q c}\right)\right)-U_{o}\left(t_{1}-t_{q f}\right)
$$

Note that the limits in equation (2.11) can be justified by considering that because of causality, $\mathrm{K}_{q}$, vanishes for $\mathrm{t}<\mathrm{t}_{q}$, and that for $t>\mathrm{t}_{\mathrm{l}}$, the integrand vanished outside the range of the window. The moments of the stochastic neutron field are given in terms of the kernel probability generating function by:

$$
n_{\{q\}}^{+}=\left(\frac{\partial^{q} G_{K}}{\partial Z_{1} \cdots \partial Z_{q}}\right)_{1}
$$

\section{THE COVARIANCE FUNCTION}

For the two detector case $\left(Q_{o}=2\right)$, the covariance function, $C_{12}(A)$, is given by

$$
{ }^{\circ} C_{12}(A)=\left\langle N_{1} N_{2}\right\rangle-\left\langle N_{1}\right\rangle\left\langle N_{2}\right\rangle
$$

where $(q=1,2)$

$$
<N_{q}>=\left(\frac{\partial G_{s}}{\partial Z_{q}}\right)_{1}
$$




$$
<N_{\mathrm{l}} N_{2}>=\left(\frac{\partial^{2} G_{s}}{\partial Z_{1} \partial Z_{2}}\right)_{1}
$$

Use of equation (2.6) yields:

$$
\begin{aligned}
<N_{q}>= & <n_{q}^{+} \mid S> \\
<N_{1} N_{2}> & =<n_{12}\left|S>+<n_{1}^{+}\right| S><n_{2}^{+} \mid S> \\
& +\overline{v_{\mathrm{s}}\left(v_{\mathrm{s}}-1\right)} \int_{-\infty}^{\infty} d t \int d \underline{r} S_{1}(\vec{r}) I_{1}\left(\vec{r}, t \mid A_{1}\right) I_{2}\left(\vec{r}, t\left(A_{2}\right)\right.
\end{aligned}
$$

where we defined the neutron source

$$
S(\vec{r}, \mathrm{v})=\bar{v}_{\mathrm{s}} \frac{\chi_{s}(\mathrm{v})}{4 \pi} S_{1}(\vec{r})\left(\frac{\text { neutrons }}{\left(\mathrm{cm}^{3}\right)(\mathrm{sec})(\text { stereorodian })(\text { unitspeed })}\right)
$$

and the source spectral factors

$$
\mathrm{Iq}(\overrightarrow{\mathrm{r}}, \mathrm{t} \mid \mathrm{Aq})=\mathrm{T}_{\mathrm{s}} n_{q}^{+}\left(1, t \mid A_{q}\right)
$$

The moments, $n_{q}^{+}$, and $n_{12}^{+}$, are given by 


$$
n_{q}^{+}=\left(\frac{\partial G_{K}}{\partial Z_{q}}\right)_{1} ; n_{12}^{+}=\left(\frac{\partial^{2} G_{K}}{\partial Z_{1} \partial Z_{2}}\right)_{1}
$$

Use of equations (3.8) and (2.8) yields the following transport equations for the $n_{q}^{+}$and $n_{12}^{+}$moments:

$$
\begin{aligned}
& \left\{-\frac{1}{\mathrm{v}} \frac{\partial}{\partial t}+L_{o}^{+}\right\} n_{q}^{+}=E_{q c} \\
& \left\{-\frac{1}{\mathrm{v}} \frac{\partial}{\partial t}+L_{o}^{+}\right\} n_{12}^{+}=Q_{12}
\end{aligned}
$$

where

$$
\begin{aligned}
& E_{q c}=\bar{\gamma}_{q} \Sigma_{o q c}(\mathrm{v}) \int_{-\infty}^{\infty} d t_{1} K_{q}\left(t-t_{1}\right) U_{q}\left(t_{1} \mid t_{q f}, \Delta_{q c}\right) \\
& Q_{12}=\overline{v_{p}\left(v_{p}-1\right)} \Sigma_{F}(\mathrm{v}) \mathrm{Y}_{1}(\vec{r}, t) \mathrm{Y}_{2}(\vec{r}, t)
\end{aligned}
$$

with

$$
\bar{\gamma}_{q}=\gamma_{q} V_{q 0}
$$

and where we introduced the spectral factors

$$
\mathrm{Y}_{q}(\vec{r}, t)=T_{R} n_{q}^{+}(1, t)
$$


The transport equations (3.9) and (3.10) are associated to the boundary conditions

$$
n q^{+}=n_{12}^{+}=0 \text { (on a convex boundary) for } \vec{\Omega} \cdot \overrightarrow{\mathrm{n}}>0
$$

In view of the structure of the adjoint operator in equations (3.9) and (3.10), the adjoint moments, $n_{q}^{+}$and $n_{12}$, satisfy time-reversed conditions, that is "final" rather than initial conditions. Clearly, because the detectors are turned-off at $t=t_{q f}$, the adjoint moments must be zero at the end of the measurements.

We now return to equation (3.1) for the covariance functions, that after inserting equations (3.4) and (3.5) becomes

$$
C_{12}(A)=\left\langle n_{12}^{+} \mid S\right\rangle+\overline{\mathrm{v}_{s}\left(\mathrm{~V}_{s-1}\right)} \int_{-\infty}^{\infty} d t \int d \underline{r} S_{1}(\vec{r}) I_{1}(\vec{r} t) I_{2}(\vec{r} t)
$$

To eliminate the adjoint moment, $n_{12}^{+}$, we introduce the neutron flux, $\phi(1, t)$, satisfying the transport equation

$$
\left(\frac{1}{\mathrm{v}} \frac{\partial}{\partial t}+L_{0}\right) \Phi=S
$$


The neutron flux is assumed to be zero in the remote past and to satisfy the boundary conditions

$$
\Phi=0 \text { (on a convex boundary) for } \vec{\Omega} \cdot \overrightarrow{\mathrm{n}}<0
$$

Use of the commutation relation (1.11) yields

$$
<n_{12}^{+}|S>=<\Phi| Q_{12}>
$$

Thus, our final result result for the covariance function is:

$$
\text { - } C_{12}(A)=<\Phi \mid Q_{12}>+\overline{\mathrm{v}_{s}\left(\mathrm{v}_{s}-1\right)} \int_{-\infty}^{\infty} d t \int d \underline{r} S_{1}(\vec{r}) I_{1}(\vec{r}, t) I_{2}(\vec{r}, t)
$$

\section{THE BICOVARIANCE FUNCTION}

For the three detector case $\left(Q_{0}=3\right)$, the Bicovariance function $C_{123}(A)$ is defined as

$$
C_{123}=\overline{\left(N_{1}-\bar{N}_{1}\right)\left(N_{2}-\bar{N}_{2}\right)\left(N_{3}-\bar{N}_{3}\right)}
$$

The calculation of the third moment, $\overline{N_{1} N_{2} N_{3}}$, involves operating with $\partial^{3} /\left(\partial Z_{1} \partial Z_{2} \partial Z_{3}\right)$ on the source probability generating function. One obtains: 


$$
\begin{aligned}
& \overline{N_{1} N_{2} N_{3}}=\overline{\mathrm{v}}_{s}\left[Q \frac{\partial^{3} F_{s}}{\partial Z_{1} \partial Z_{2} \partial Z_{3}}\right] \\
& +\overline{\mathrm{v}_{s}\left(\mathrm{v}_{s}-1\right)}\left[Q\left\{\left(\frac{\partial^{3} F_{s}}{\partial Z_{1} \partial Z_{2}}\right) \frac{\partial F_{s}}{\partial Z_{3}}+\left(\frac{\partial^{2} F_{s}}{\partial Z_{1} \partial Z_{3}}\right) \frac{\partial F_{s}}{\partial Z_{2}}+\left(\frac{\partial^{2} F_{s}}{\partial Z_{2} \partial Z_{3}}\right) \frac{\partial F_{s}}{\partial Z_{1}}\right\}\right] \\
& +\left(\overline{\mathrm{v}}_{s}\right)^{2}\left\{\left[Q \frac{\partial^{2} F_{s}}{\partial Z_{1} \partial Z_{2}}\right]\left[Q \frac{\partial F_{s}}{\partial Z_{3}}\right]+\left[Q \frac{\partial^{2} F_{s}}{\partial Z_{1} \partial Z_{3}}\right]\left[Q \frac{\partial F_{s}}{\partial Z_{2}}\right]+\left[Q \frac{\partial^{2} F_{s}}{\partial Z_{2} \partial Z_{3}}\right]\left[Q \frac{\partial F_{s}}{\partial Z_{1}}\right]\right\} \\
& +\overline{\mathrm{v}_{s}} \cdot \overline{\mathrm{v}_{s}\left(\mathrm{v}_{s}-1\right)}\left\{Q\left[\frac{\partial F_{s}}{\partial Z_{1}} \frac{\partial F_{s}}{\partial Z_{2}}\right]\left[Q \frac{\partial F_{s}}{\partial Z_{3}}\right]+\left[Q \frac{\partial F_{s}}{\partial Z_{1}} \frac{\partial F_{s}}{\partial Z_{3}}\right]\left[Q \frac{\partial F_{s}}{\partial Z_{2}}\right]+\left[Q \frac{\partial F_{s}}{\partial Z_{2}} \frac{\partial F_{s}}{\partial Z_{3}}\right]\left[Q \frac{\partial F_{s}}{\partial Z_{1}}\right]\right\} \\
& +\overline{\mathrm{v}_{s}\left(\mathrm{v}_{s}-1\right)\left(\mathrm{v}_{s}-2\right)}\left\{Q\left[\frac{\partial F_{s}}{\partial Z_{1}} \frac{\partial F_{s}}{\partial Z_{2}} \frac{\partial F_{s}}{\partial Z_{3}}\right]\right\} \\
& +\left(\overline{\mathrm{v}}_{s}\right)^{3}\left[Q \frac{\partial F_{s}}{\partial Z_{1}}\right]\left[Q \frac{\partial F_{3}}{\partial Z_{2}}\right]\left[Q \frac{\partial F_{s}}{\partial Z_{3}}\right]
\end{aligned}
$$

where all derivatives in (4.2) are evaluated at $Z_{1}=Z_{2}=Z_{3}=1$. The partial derivatives in equation (4.2) are calculated from the relationship (2.8) one obtains: $(q=1,2,3)$

$$
\left(\frac{\partial F_{s}}{\partial Z_{q}}\right)_{1}=T_{s} n_{q}^{+},\left(\frac{\partial^{2} F_{s}}{\partial Z_{q} \partial Z_{q^{\prime}}}\right)=T_{s} n_{q}^{+}{ }_{q^{\prime}}\left(q \neq q^{\prime}\right)
$$


and

$$
\left(\frac{\partial^{3} F_{s}}{\partial Z_{1} \partial Z_{2} \partial Z_{3}}\right)_{1}=T_{s} n_{123}^{+}
$$

where the adjoint moments are defined by the relations

$$
n_{q}^{+}=\left(\frac{\partial G_{K}}{\partial Z_{q}}\right)_{1} ; n_{q q^{\prime}}^{+}=\left(\frac{\partial^{2} G_{K}}{\partial Z_{q} \partial Z_{q^{\prime}}}\right) ; n_{123}^{+}=\left(\frac{\partial^{3} G_{K}}{\partial Z_{1} \partial Z_{2} \partial Z_{3}}\right)
$$

Insertion of the above results into (4.2) yields.

$$
\begin{aligned}
\overline{N_{1} N_{2} N_{3}} & =<n_{123}^{+} \mid S>+\overline{\mathrm{v}_{\mathrm{s}}\left(\mathrm{v}_{\mathrm{s}}-1\right)} Q\left[I_{12} I_{3}+I_{13} I_{2}+I_{23} I_{1}\right] \\
& +<n_{12}^{+}\left|S><n_{3}^{+}\right| S>+<n_{13}^{+}\left|S><n_{2}^{+}\right| S>+<n_{23}^{+}\left|S><n_{1}^{+}\right| S> \\
& +\overline{\mathrm{v}_{s}\left(\mathrm{v}_{s}-1\right)}\left\{\left[Q I_{1} I_{2}\right]<n_{3}^{+}\left|S>+\left[Q I_{1} I_{3}\right]<n_{2}^{+}\right| S>+\left[Q I_{2} I_{3}\right]<n_{1}^{+} \mid S>\right\} \\
& +\overline{\mathrm{v}_{s}\left(\mathrm{v}_{s}-1\right)\left(\mathrm{v}_{s}-2\right)}\left[Q I_{1} I_{2} I_{3}\right]+<n_{1}^{+}\left|S><n_{2}^{+}\right| S><n_{3}^{+} \mid S>(4.6)
\end{aligned}
$$

where we introduced the neutron source, S, equation (3.6), the spectral factors (3.7), and the two-detector spectral factors

$$
I_{q q^{\prime}}(\vec{r})=T_{s} n_{q q^{\prime}}^{+}=I_{q^{\prime} q}
$$

Similarly, one obtains $\left(q \neq q^{\prime}\right)$ 


$$
\overline{N_{q} N_{q^{\prime}}}=\overline{N_{q^{\prime}} N_{q}}=<n_{q q^{\prime}}^{+} \mid S>+\overline{\mathrm{v}_{s}\left(\mathrm{v}_{s}-1\right)} Q\left[I_{q} I_{q^{\prime}}\right]+\left\langle n_{q}^{+}\right| S>\left\langle n_{q^{\prime}}^{+} \mid S\right\rangle
$$

Insertion of equations (4.6) and (4.8) into equation (4.1) for the bicovariance function yields:

$$
\begin{aligned}
C_{\mathrm{l} 23}\left(A_{3}\right) & =<n_{123}^{+} \mid S>+\overline{\mathrm{v}_{s}\left(\mathrm{~V}_{s}-1\right)} Q\left\{I_{12} I_{3}+I_{13} I_{2}+I_{23} I_{1}\right\} \\
& +\overline{\mathrm{v}_{s}\left(\mathrm{v}_{s}-1\right)\left(\mathrm{v}_{s}-2\right)} Q\left[I_{1} I_{2} I_{3}\right]
\end{aligned}
$$

The evolution equations for the adjoint moments, $n_{q}^{+}$and $n_{q q^{\prime}}^{+}$, were given in section three. The newly obtained third order adjoint moment, $n_{123}^{+}$, is determined by the equation

$$
\begin{aligned}
\left\{-\frac{1 \partial}{\mathrm{v} \partial t}+L_{o}^{+}\right\} n_{123}^{+} & =\overline{v_{p}\left(v_{p}-1\right)}\left[\mathrm{Y}_{12} \cdot \mathrm{Y}_{3}+\mathrm{Y}_{13} \mathrm{Y}_{2}+\mathrm{Y}_{23} \mathrm{Y}_{1}\right] \Sigma_{F} \\
& +\overline{v_{p}\left(v_{p}-1\right)\left(v_{p}-2\right)} \mathrm{Y}_{1} \mathrm{Y}_{2} \mathrm{Y}_{3} \Sigma_{F}
\end{aligned}
$$

where the two-detector spectral factors, $Y_{q q^{\prime}}$, were introduced

$$
\mathrm{Y}_{q q^{\prime}}=T_{R} n_{q q^{\prime}}^{+}=\mathrm{Y}_{q^{\prime} q}
$$


and use was made of equations (4.5) and (4.3). Note that the third order adjoint moments satisfy the same boundary and final conditions that the lower rank moments, $n_{q}^{+}$and $n_{q q^{\prime}}^{+}$. To eliminate the adjoint moment, $n_{123}^{+}$, from equation (4.9), we use equation (3.17) for the neutron flux and the commutation relation (1.11) to obtain:

$$
\begin{aligned}
C_{123}\left(A_{3}\right) & =\overline{v_{p}\left(v_{p}-1\right)}<\Phi \mid \Sigma_{F}\left(\mathrm{Y}_{12} \mathrm{Y}_{3}+\mathrm{Y}_{13} \mathrm{Y}_{2}+\mathrm{Y}_{23} \mathrm{Y}_{1}\right)> \\
& +\overline{v_{p}\left(v_{p}-1\right)\left(v_{p}-2\right)}<\Phi \mid \Sigma_{F} \mathrm{Y}_{1} \mathrm{Y}_{2} \mathrm{Y}_{3}> \\
& +\overline{v_{s}\left(v_{s}-1\right)} Q\left\{I_{12} I_{3}+I_{13} I_{2}+I_{23} I_{1}\right\} \\
& +\overline{v_{s}\left(v_{s}-1\right)\left(v_{s}-2\right)} Q_{I_{1} I_{2} I_{3}}
\end{aligned}
$$

\section{THE TWO AND THREE DETECTORS CROSS CORRELATION FUNCTIONS}

In practice, the noise description used in the cross correlation function which is defined in terms of instantaneous count rates, as measured by a time analyzer during a record length of $t_{q F}$ seconds. This device opens a sequence of gates (channels) of duration much shorter than the total record length. The counts stored in each channel is the count rate, with a time resolution fixed by the channel width.

We then define the two and three detector cross correlation functions as the limits 


$$
\begin{gathered}
\Phi_{12}\left(t_{1 f_{1}} t_{2 f}\right)=\underset{\Delta_{q c \rightarrow o}}{\operatorname{limit}}\left\{\frac{C_{12}\left(A_{2}\right)}{\Delta_{1 c} \Delta_{2 c}}\right\} \\
\Phi_{12}\left(t_{1 f}, t_{2 f}, t_{3 f}\right)=\operatorname{limit}_{\Delta_{q c \rightarrow 0}}\left\{\frac{C_{12}\left(A_{2}\right)}{\Delta_{1 c} \Delta_{2 c}}\right\}
\end{gathered}
$$

After carrying out the indicated limits in equations (3.20), (4.9), and (3.7), (3.14), (3.9), (3.10), and (3.12), one obtains

$$
\Phi_{12}\left(\tau_{1}\right)=\Phi_{12 s y s}\left(\tau_{1}\right)+\Phi_{12 s}\left(\tau_{1}\right)
$$

with

$$
\begin{aligned}
& \Phi_{12 s y s}=\overline{v_{p}\left(v_{p}-1\right)}<\Phi \mid \Sigma_{F} \Theta_{1} \Theta_{2}> \\
& \Phi_{12 s}=\overline{v_{s}\left(v_{s}-1\right)} \int_{-\infty}^{\infty} d t \int d r S_{1}(\vec{r}) \mathrm{J}_{1}(\vec{r}, t) \mathrm{J}_{2}(\vec{r}, t) \\
& \Phi_{123}\left(\tau_{1}, \tau_{2}\right)=\Phi_{123 \text { sss }}\left(\tau_{1}, \tau_{2}\right)+\Phi_{123 s}\left(\tau_{1}, \tau_{2}\right)
\end{aligned}
$$

with 


$$
\begin{aligned}
\Phi_{123 s y s}= & \overline{v_{p}\left(v_{p}-1\right)\left(v_{p}-2\right)}<\Phi \mid \Sigma_{F} \Theta_{1} \Theta_{2} \Theta_{3}> \\
& +\overline{v_{p}\left(v_{p}-1\right)}<\Phi \mid \Sigma_{F}\left(\Theta_{12} \Theta_{3}+\Theta_{13} \Theta_{2}+\Theta_{13} \Theta_{1}\right)> \\
\Phi_{123 s}= & \overline{v_{s}\left(v_{s}-1\right)\left(v_{s}-2\right)} \int_{-\infty}^{\infty} d t \int d \underline{r} S_{1}(\vec{r}) \mathrm{J}_{1} \mathrm{~J}_{2} \mathrm{~J}_{3} \\
& +\overline{v_{s}\left(v_{s}-1\right)} \int_{-\infty}^{\infty} d t \int d \underline{r} S_{1}(\vec{r})\left(\mathrm{J}_{12} \mathrm{~J}_{3}+\mathrm{J}_{13} \mathrm{~J}_{2}+\mathrm{J}_{23} \mathrm{~J}_{1}\right)
\end{aligned}
$$

where we introduced the delay times

$$
\tau_{1}=t_{F 2}-t_{F 1} ; \tau_{2}=t_{F 3}-t_{F 2}
$$

where

$$
\Theta_{q}=\underset{\Delta_{q c \rightarrow 0}}{\operatorname{limit}}\left\{\frac{\mathrm{Y}_{\mathrm{q}}}{\Delta_{\mathrm{qc}}}\right\}=T_{R} p_{q}^{+}
$$

$$
\Phi_{q q^{\prime}}=\operatorname{limit}_{\Delta_{q c}, \Delta_{q^{\prime} c \rightarrow 0}}\left\{\frac{\mathrm{Y}_{q q^{\prime}}}{\Delta_{q c} \Delta_{q^{\prime} c}}\right\}=T_{R} p_{q q^{\prime}}^{+}
$$

16 


$$
\mathrm{J}_{q}=\operatorname{limit}_{\Delta_{q c \rightarrow 0}}\left\{\frac{I_{q}}{\Delta_{\mathrm{qc}}}\right\}=T_{s} p_{q}^{+}
$$

$$
\begin{aligned}
& \mathrm{J}_{q q^{\prime}}=\operatorname{limit}_{\Delta_{q c}}, \Delta_{q^{\prime} c \rightarrow 0}\left\{\frac{I_{q q^{\prime}}}{\Delta_{q c} \Delta_{q^{\prime} c}}\right\}=T_{s} p_{q q^{\prime}}^{+} \\
& p_{q}^{+}=\operatorname{limit}_{\Delta_{q c \rightarrow 0}}\left\{\frac{n_{q}^{+}}{\Delta_{q c}}\right\}
\end{aligned}
$$

$$
p_{q q^{\prime}}^{+}=\operatorname{limit}_{\Delta_{\mathrm{qc}}} \Delta_{q^{\prime} c}\left\{\frac{\mathrm{n}_{\mathrm{qq}}^{+}}{\Delta_{\mathrm{qc}} \Delta_{q^{\prime} c}}\right\}
$$

The new adjoint moments, $p_{q}^{+}$and $p_{q q^{\prime}}^{+}$, satisfy the same boundary and final conditions than the old ones and the equations

$$
\left\{-\frac{1}{\mathrm{v}} \frac{\partial}{\partial t}+L_{o}^{+}\right\} p_{q}^{+}=D_{q c}
$$




$$
\left\{-\frac{1}{\mathrm{v}} \frac{\partial}{\partial t}+L_{o}^{+}\right\} p_{q q^{\prime}}^{+}=M_{q q^{\prime}}
$$

where

$$
\begin{aligned}
& D_{q c}=\operatorname{limit}_{\Delta_{\mathrm{qc} \rightarrow 0}}\left\{\frac{\mathrm{E}_{\mathrm{qc}}}{\Delta_{q c}}\right\}=\bar{\gamma}_{q} K_{q}\left(t-t_{q F}\right) \Sigma_{o q c}(\mathrm{v}) \delta\left(\vec{r}-\vec{r}_{q}\right) \\
& M_{q q^{\prime}}=\operatorname{limit}_{\Delta_{\mathrm{qc}}, \Delta_{q^{\prime} c \rightarrow o}}^{\operatorname{lig}}\left\{\frac{\mathrm{Q}_{12}}{\Delta_{\mathrm{qc}} \Delta_{q^{\prime} c}}\right\}=\overline{v_{p}\left(v_{p}-1\right)} \Sigma_{F} \Theta_{q} \Theta_{q^{\prime}}
\end{aligned}
$$

The moments, $p_{q}^{+}$, driven by the detector location and strength are generalized functions having the physical meaning of a detector field-of-view function. These functions peak at the detector location and are time-displacement kernels. Because of the definition (5.10), the quantities, $\Theta_{q}, \mathrm{~J}_{q}$, are velocity and angle averaged detector field-of-view functions. The former are averaged over the fission neutron spectrum of the fissile material where the $\mathrm{J}_{\mathrm{q}}$-quantities are averaged over the source fission spectrum.

In writing the result (5.3) for the two-detector cross correlation function, the subindex $(1,2)$ indicates that the detector 2 is delayed by a time, $\tau_{1}$, with respect to detector 1 . Clearly, $\Phi_{12}\left(\tau_{1}\right)=\Phi_{21}\left(-\tau_{1}\right)$. 
The result (5.3) shows that the two-detector cross correlation function has a component driven by the inherent fluctuation of the fission process in the fissile material and a second component directly depending on the source fluctuations. Correlation contributions occur when the field-of-view of the detectors overlap in space and there is a delayed overlap in the time domain by, $\tau_{1}$, units.

In regard to the three-detector cross correlation functions, the $(1,2,3)$ notation indicates that detector 2 is delayed by a time, $\tau_{1}$, and detector 3 by a time $\tau_{2}$, both with respect to the first detector.

Note that the $p_{4 q^{\prime}}^{+}$, adjoint moments, equation (5.16) are driven by the induced fissions in the system weighted by the overlapping of the $\left(q q^{\prime}\right)$ pair of velocity and angle weighted detector field-of-view functions, $\Theta_{q}$ and $\Theta_{q^{\prime}}$. One can portray these generalized functions as the field-of-view for these fission chains born at the $\vec{r}$ location that were detected with delayed time overlaps later by the $\left(q, q^{\prime}\right)$ detectors. The first term on the right hand side of equations (5.7) and (5.8) are the extension to the three detector case of the $\Phi_{12 \text { sys }}$ and $\Phi_{125}$ terms in the two-detector cross correlation case. Both terms are proportional to the third moment of their respective neutron multiplicity distribution functions. 
The second term on the right hand side of equation (5.7) is a new one that is given as the sum of three terms each one consisting of the product of a $\Theta_{q q^{\prime}}\left(q \pm q^{\prime}\right)$, factor

representing the $\left(q q^{\prime}\right)$ field-of-view of the fission chain and a factor $\Theta_{q^{\prime \prime}}\left(q^{\prime \prime} \neq q, q^{\prime}\right)$ representing the field-of-view of the $q^{\prime \prime}-t h$ detector. From equations $5.11,5.16$, and 5.18 , one realizes that this term is proportional to the square of the fission cross section. Finally, the second term on the right hand side of equation 5.8 exhibits the same structure as the previously discussed term, but it is only linearly dependent on the fission cross section. Also not that the three-detector cross correlation function is asymmetric with respect to the detector taken as the reference for zero delay.

\section{THE BISPECTRUM FUNCTION}

To derive an expression for the bispectrum function operate on equation (5.6) with

$$
\begin{gathered}
(2 \pi)^{-2} \int_{-\infty}^{\infty} d \tau_{1} d \tau_{2} \exp \left\{i\left(\omega_{1} \tau_{1}+\omega_{2} \tau_{2}\right\}\right. \\
\left(\tau_{1}=t_{2 f}-t_{1 f} ; \tau_{2}=t_{3 f}-t_{1 f}\right)
\end{gathered}
$$

and follow the steps in Appendix A to obtain: 


$$
\begin{aligned}
\Phi_{123 s y s}\left(\omega_{1} \omega_{2}\right) & =\overline{v_{p}\left(v_{p}-1\right)\left(v_{p}-2\right)} B_{15 y s}\left(\omega_{1} \omega_{2}\right) \\
& +\overline{v_{p}\left(v_{p}-1\right)} B_{2 s y s}\left(\omega_{1} \omega_{2}\right) \\
\Phi_{s}\left(\omega_{1} \omega_{2}\right) & =\overline{v_{s}\left(v_{s}-1\right)\left(v_{s}-2\right)} B_{1 s}\left(\omega_{1} \omega_{2}\right) \\
& +\overline{v_{s}\left(v_{s}-1\right)} B_{2 s}\left(\omega_{1} \omega_{2}\right)
\end{aligned}
$$

where we introduced the bispectrum functions

$$
\begin{aligned}
B_{1 s y s}= & (2 \pi)^{-2}\left(\Phi_{s} \mid \Sigma_{F} \Theta_{1}\left(\omega_{1}+\omega_{2}\right) \Theta_{2}^{*}(\omega) \Theta_{3}^{*}\left(\omega_{2}\right)\right) \\
B_{1 s}= & (2 \pi)^{-2} \int d \underline{r} S_{1}(\vec{r}) \mathrm{J}_{1}\left(\vec{r}, \omega_{1}+\omega_{2}\right) \mathrm{J}_{2}\left(\vec{r}, \omega_{1}\right) \mathrm{J}_{3}\left(\vec{r}, \omega_{2}\right) \\
B_{2 S Y S}= & (2 \pi)^{-2}\left(\Phi_{s} \mid \Sigma_{F}\left[\Theta_{13}\left(\left[\omega_{1}+\omega_{2}\right]-\omega_{1}\right) \Theta_{3}^{*}\left(\omega_{2}\right)+\right.\right. \\
& +\Theta_{13}\left(\left[\omega_{1}+\omega_{2}\right]-\omega_{2}\right) \Theta_{2}^{*}\left(\omega_{1}\right)+ \\
& \left.\left.+\Theta_{23}^{*}\left(\omega_{1} \omega_{2}\right) \Theta_{1}\left(\omega_{1}+\omega_{2}\right)\right]\right) \\
B_{2 s}= & (2 \pi)^{-2} \int d \underline{r} S_{1}(\vec{r})\left[\mathrm{J}_{12}\left(\left(\omega_{1}+\omega_{2}\right)-\omega_{1}\right) \mathrm{J}_{3}^{*}\left(\omega_{2}\right)\right. \\
& +\mathrm{J}_{13}\left(\left[\omega_{1}+\omega_{2}\right]-\omega_{2}\right) \mathrm{J}_{2}^{*}\left(\omega_{1}\right) \\
& \left.\left.\left.+\mathrm{J}_{23}^{*}\left(\omega_{1}, \omega_{2}\right) \mathrm{J}_{1}\left(\omega_{1}+\omega_{2}\right)\right]\right)\right]
\end{aligned}
$$


Insertion of the results (B.5), (B.6), (B.10) and (B.11) yields:

$$
\begin{aligned}
\Phi_{123 s y s} & =M_{123}\left(\omega_{1} \omega_{2}\right) \overline{\left(v_{p}\left(v_{p}-1\right)\left(v_{p}-2\right)\right.} b_{1 s y s}\left(\omega_{1} \omega_{2}\right) \\
& \left.+\left(\overline{v_{p}\left(v_{p}-1\right.}\right)^{2} b_{2 s y s}\left(\omega_{1} \omega_{2}\right)\right\} \\
\Phi_{123 s} & =M_{123}\left(\omega_{1} \omega_{2}\right)\left\{\overline{v_{s}\left(v_{s}-1\right)\left(v_{s}-2\right)} b_{15}\left(\omega_{1} \omega_{2}\right)\right. \\
& \left.+\overline{v_{p}\left(v_{p}-1\right)} \overline{v_{s}\left(v_{s}-1\right)} b_{2 s}\left(\omega_{1} \omega_{2}\right)\right\}
\end{aligned}
$$

with

$$
\begin{aligned}
M_{123}=(2 \pi)^{-2} \bar{\gamma}_{1} \bar{\gamma}_{2} \bar{\gamma}_{3} K_{1}\left(\omega_{1}+\omega_{2}\right) K_{2}^{*}\left(\omega_{1}\right) K_{3}^{*}\left(\omega_{2}\right) \\
b_{1 s y s}=\left(\Phi_{s} \mid \Sigma_{F} h_{R_{1}}\left(\omega_{1}+\omega_{2}\right) h_{R 2}\left(\omega_{1}\right) h_{R 3}\left(\omega_{2}\right)\right) \\
b_{1 s=} d \underline{r} S_{1}(\vec{r}) h_{s 1}\left(\omega_{1}+\omega_{2}\right) h_{s 2}^{*}\left(\omega_{1}\right) h_{s 3}^{*}\left(\omega_{2}\right) \\
b_{2 s y s}=\left(\Phi_{s} \mid \Sigma_{F}\left[h_{R 12}\left(\left[\omega_{1}+\omega_{2}\right]-\omega_{1}\right) h_{R 3}^{*}\left(\omega_{2}\right)\right.\right. \\
+h_{R 13}\left(\left[\omega_{1}+\omega_{2}\right]-\omega_{2}\right) h_{R 2}^{*}\left(\omega_{1}\right) \\
+
\end{aligned}
$$




$$
\begin{aligned}
& b_{2 s}=\int d \underline{r} S_{1}(r)\left[h_{s 12}\left(\left[\omega_{1}+\omega_{2}\right]-\omega_{1}\right) h_{s 3}^{*}\left(\omega_{2}\right)\right. \\
& +h_{s 13}\left(\left[\omega_{1}+\omega_{2}\right]-\omega_{2}\right)^{*} h_{s 2}^{*}\left(\omega_{1}\right) \\
& \left.+h_{s 23}^{*}\left(\omega_{1} \omega_{2}\right) h_{s 1}^{\prime}\left(\omega_{1}+\omega_{2}\right)\right]
\end{aligned}
$$

Note that by going to the frequency domain the convolution integrals transform into products that result in the separation of the instrumentation and system responses.

The various $h_{q}$-functions defined in Appendix B, are Fourier, transformed detector fieldof-view functions whereas the $h_{q q^{\prime}}$-functions are driven by the space overlap of the $\left(q, q^{\prime}\right)$ detector pair over the distribution of the fissile material.

\section{THE SINGLE MODE APPROXIMATION}

In some instances, specially for compact systems exhibiting simple geometries, the single mode representation (one-point reactor kinetics) is an adequate description of the system behavior. Start from the representation of the propagator in terms of the fundamental eigenmode, ( $n=0$, see Appendices $B$ and $C)$.

$$
G(1 \mid 2 \omega)=G_{o}(\omega) \Phi_{o}(1) \Phi_{o}^{+}(2)
$$

Insertion of (7.1) into equation B.2 for the Fourier transformed propagator yields, upon use of the biorthogonal relations (C.4) and equation (C.9). 


$$
G_{o}\left(\omega_{1}\right)=\frac{1}{N_{o o}}(\alpha+i \omega)^{-1}
$$

where to follow standard ways we defined the Rossi- $\alpha$ as

$$
\alpha=\left|\omega_{o}\right|
$$

Insertion of (7.1) into equations (B.7), (B.8), (B.10), and (B.11) yields

$$
\begin{aligned}
& h_{R q}(\vec{r}, \omega)=C_{q} W_{R}(\vec{r}) g(\omega) \\
& h_{S q}(\vec{r}, \omega)=C_{q} W_{S}(\vec{r}) g(\omega) \\
& h_{R q q^{\prime}}\left(\vec{r} \omega_{1} \omega_{2}\right)=C_{q} C_{q^{\prime}}\left(\Phi_{o} \mid \Sigma_{F} W_{R}^{2}\right) W_{R}(\vec{r}) g\left(\omega_{1}\right) g\left(\omega_{2}\right) g\left(\omega_{1} \omega_{2}\right)(7.6) \\
& h_{S q q^{\prime}}\left(\vec{r} \omega_{1} \omega_{2}\right)=C_{q} C_{q^{\prime}}\left(\Phi_{o} \mid \Sigma_{F} W_{S}^{2}\right) W_{S}(\vec{r}) g\left(\omega_{1}\right) g\left(\omega_{2}\right) g\left(\omega_{1} \omega_{2}\right)(7.7)
\end{aligned}
$$

where

$$
C_{q R}=\text { Capture rate in the qth detector }=j d v d \Omega \Sigma_{o q c}(\mathrm{v}) \Phi_{o}\left(\vec{r}_{q}, \mathrm{v} \vec{\Omega}\right)
$$




$$
g(\omega)=\text { First order transfer function }=(\alpha+\mathrm{i} \omega)^{-1}
$$

and where we introduced the weights

$$
\begin{aligned}
& W_{R}(\vec{r})=N_{o o}^{-1} \int d \mathrm{v} d \Omega \frac{\chi(\mathrm{v})}{4 \pi} \Phi_{o}^{+}(\vec{r}, \mathrm{v}, \vec{\Omega}) \\
& W_{S}(\vec{r})=N_{o o}^{-1} \int d \mathrm{v} d \Omega \frac{\chi_{s}(\mathrm{v})}{4 \pi} \Phi_{o}^{+}(\vec{r}, \mathrm{v}, \vec{\Omega})
\end{aligned}
$$

Define now the reduced bispectrum functions

$$
\varphi_{123 \text { sys }}=\Phi_{123 \text { sys }} / M_{123} ; \varphi_{123}=\Phi_{123 s} / M_{123}
$$

and use equations (7.4) up to (7.7.) to obtain

$$
\begin{aligned}
& \left.\varphi_{123}\left(\omega_{1} \omega_{2}\right)=\left(\Phi_{o} \mid \Sigma_{F} W_{R}^{3}\right) \overline{\left(v_{p}\left(v_{p}-1\right)\left(v_{p}-2\right)\right.}+\eta_{1} \overline{v_{s}\left(v_{s}-1\right)\left(v_{s}-2\right)}\right\} T_{1}\left(\omega_{1} \omega_{2}\right) \\
& {\left[\left(\Phi_{o} \mid \Sigma_{F} W_{R}^{2}\right)\right]^{2} \overline{v_{p}\left(v_{p}-1\right)}\left\{\overline{v_{p}\left(v_{p}-1\right)}+\eta_{2} \overline{v_{s}\left(v_{s}-1\right)}\right\} T_{2}\left(\omega_{1} \omega_{2}\right)(7.13)}
\end{aligned}
$$

with 


$$
\begin{aligned}
& \eta_{1}=\frac{\left(S_{1} \mid \omega_{S}^{3}\right)}{\left(\Phi_{O} \mid \Sigma_{F} \omega_{R}^{3}\right)} \text { (dimensionless) } \\
& \eta_{2}=\frac{\left(S_{1} \mid \omega_{S}^{2}\right)}{\left(\Phi_{o} \mid \Sigma_{F} \omega_{R}^{2}\right)} \text { (dimensionless) } \\
& T_{1}\left(\omega_{1} \omega_{2}\right)=C_{1 R} C_{2 R} C_{\dot{3} R} g\left(\omega_{1}+\omega_{2}\right) g^{*}\left(\omega_{1}\right) g^{*}\left(\omega_{2}\right) \\
& T_{2}\left(\omega_{1} \omega_{2}\right)=\left[g\left(\omega_{1}\right)+g\left(\omega_{2}\right)+g\left(\omega_{1}+\omega_{2}\right)\right] T_{1}\left(\omega_{1} \omega_{2}\right)
\end{aligned}
$$

The bispectrum, $T_{\mathrm{f}}$, can be rewritten in the form:

$$
T_{1}\left(\omega_{1} \omega_{2}\right)=C_{1 R} C_{2 R} C_{3 R}\left(\mu_{o}+i v_{o} / W_{o}^{2}\right)
$$

with

$$
\begin{aligned}
& \mu_{o}=\alpha\left[\omega_{1}^{2}+\omega_{2}^{2}+\alpha^{2}+\omega_{1} \omega_{2}\right] \\
& \nu_{o}=\omega_{1} \omega_{2}\left(\omega_{1}+\omega_{2}\right) ; \mathrm{W}_{o}^{2}=\mu_{o}^{2}+V_{o}^{2}
\end{aligned}
$$

whereas the $T_{2}$ - response function is given by 


$$
\begin{aligned}
T_{2 R}\left(\omega_{1} \omega_{2}\right) & =C_{1 R} C_{2 R} C_{3 R} \dot{W}_{o}^{-2}\left\{\frac{\mu_{o} \alpha+\nu_{o} \omega_{1}}{\alpha^{2}+\omega_{1}^{2}}+\frac{\mu_{o} \alpha+\nu_{o} \omega_{2}}{\alpha^{2}+\omega_{2}^{2}}\right. \\
& \left.+\frac{\mu_{o} \alpha+v_{o}\left(\omega_{1}+\omega_{2}\right)}{\alpha^{2}+\left(\omega_{1}+\omega_{2}\right)^{2}}\right\} \\
T_{2 I}\left(\omega_{1} \omega_{2}\right) & =C_{1 R} C_{2 R} C_{3 R} W_{o}^{-2}\left\{\frac{\nu_{o} \alpha-\mu_{o} \omega_{1}}{\alpha^{2}+\omega_{1}^{2}}+\frac{\nu_{o} \alpha-\mu_{o} \omega_{2}}{\alpha^{2}+\omega_{2}{ }^{2}}\right. \\
& \left.+\frac{v_{o} \alpha-\mu_{o}\left(\omega_{1}+\omega_{2}\right)}{\alpha^{2}+\left(\omega_{1}+\omega_{2}\right)^{2}}\right\}
\end{aligned}
$$

Note that by starting from a rigorous derivation of the bispectrum function in terms of the stochastic theory of the neutron field, the single mode approximation provides weight factors, $W_{R}, W_{S}$, to properly account for the averaging over phase-space implicit in the one-point reactor kinetics model. The neutron source, $\mathrm{S}_{1}$, is weighted by the source neutron spectrum, $\chi_{s}(\mathrm{v})$, and the fission density by the fuel neutron spectrum, $\chi(\mathrm{v})$.

The result (7.13) was used to calculate the bispectrum obtained from a Cf-source driven noise field in an unreflected $15-\mathrm{cm}$ radius sphere of $93.15 \%$ enriched uranium metal. The Monte-Carlo code MCNP.DS ${ }^{6}$ was used to calculate the Rossi- $\alpha$ by tallying the distribution of times between ${ }^{252} \mathrm{Cf}$ fission events and system events. The evolution of the time correlated counts was well represented by an exponential dependence on the delay time proving the adequacy of the single mode representation. A fit to the correlated count 
rate yielded a Rossi- $\alpha=26.78(\mu \mathrm{s})^{-1}$. Table I displays the moments of the neutron multiplicity distribution $\mathrm{P}(\mathrm{v})$, for the Californium source and for the fissile system.

The resulting bispectrum is shown in Figure 1. As expected the intersection of the bispectrum function with the $\omega_{1}=0, \omega_{2}=0, \omega_{1}=\omega_{2}$, planes, exhibit the shape of the transfer function of a first order system having a break frequency of $\alpha$-radians.

\section{SUMMARY AND DISCUSSION}

On the basis of the general stochastic neutron field theory developed by Munoz-Cobo et al, ${ }^{5}$ results on the covariance and bicovariance of the neutron field have been presented. These two statistical quantities are obtained from the counts observed in detectors operating during a period of time (gate length), $\Delta_{\mathrm{qc}}$. A classical example is the so called Feynmann Y-function that is defined as the variance to mean ratio of the neutron field (see for instance reference 5). Upon taking the limit of the covariance and bicovariance function for $\Delta_{q c} \rightarrow o$, one obtains the two and three detector cross correlation functions respectively. The mathematical structure of the results so obtained have a transparent physical interpretation in terms of the space and delay time overlap between the field-ofview of the detectors. For the first time, an expression has been obtained for the bispectrum function of the stochastic neutron field and for the appropriate weight functions to be used as space-energy-angle correction factors for the one-point kinetics approximation. 
Table 1

\section{MOMENTS OF THE NEUTRON MULTIPLICITY DISTRIBUTION FOR THE CF-SOURCE AND THE SYSTEM}

\begin{tabular}{ll}
\multicolumn{1}{c}{ Cf-252 $^{\mathbf{a}}$} & \multicolumn{1}{c}{ System $^{\mathbf{b}}$} \\
\hline $\bar{v}=3.773$ & $\bar{v}=2.605$ \\
$\overline{v(v-1)}=12.042$ & $\overline{v(v-1)}=5.516$ \\
$\overline{v(v-1)(v-2)}=31,962$ & $\overline{v(v-1)(v-2)}=9.328$ \\
\hline
\end{tabular}

${ }^{\mathrm{a}}$ Calculated from Spencer Data. ${ }^{13}$

${ }^{b}$ Calculated by tallying fission neutron multiplicity to obtain, $\mathrm{P}(v)$, for the system driven by the $\mathrm{Cf}$-source. 


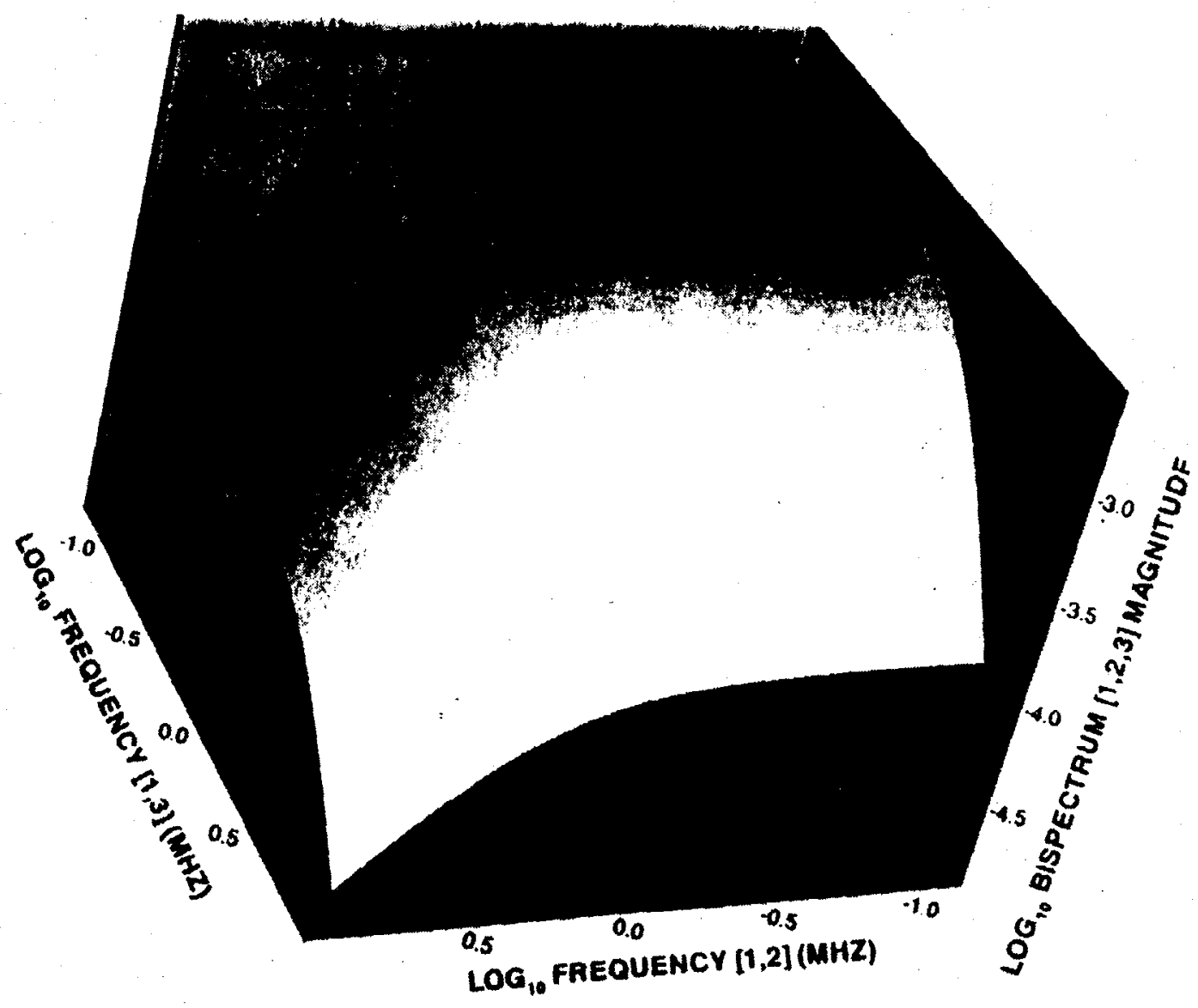

Fig. 1. Bispectrum From a Cf-Source Driven Noise Field in an Unreflected Uranium 235-Sphere. 


\section{REFERENCES}

1. Serber, R., The Defintion of "Neutron Multiplication," USAEC Report LA-335 (Rev.), Los Alamos Scientific Laboratory, July, 1945.

2. Feynmann, R. P., DeHoffman, F., and Serber, R., "Dispersion of the Neutron Emission in U-235 Fission," Journal of Nuclear Energy, Vol. 3, p. 64, 1956.

3. Orndoff, J. D., "Prompt Neutron Periods of Metal Critical Assemblies," Nuclear Science and Engineering, Vol. 2, p. 450, 1957.

4. Bell, G. I., "On the Stochastic Theory of Neutron Transport," Nuclear Science and Engineering, Vol. 21, p. 390,. 1965.

5. Munoz-Cobo, J. L., Perez, R. B., and Verdu, G., Nuclear Science and Engineering, Vol. 95, p. 83-105, 1987.

6. Valentine, T. E., and Mihalczo, J. T., "MNCP-DSP: A Neutron and Gamma Ray Monte Carlo Calculation of Source-Driven Noise-Measured Parameters," Ann. Nucl. Energy, 23, 16, 1271 (1996).

7. Mihalczo, J. T., Trans. Am. Nucl. Soc. 9 , 175 (1966).

8. Mihalczo, J. T., Pare, V. K., Ragan, G. L., Mathis, M. V., and Tillett, G. C., Nucl. Sci. \& Eng. 66, 29-59 (1978).

9. Mihalczo, J. T., and Valentine, T. E., Fifth International Conference on Nuclear Criticality Safety, September 17-21, Albuquerque, New Mexico (1995).

10. Mihalczo, J. T., and Valentine, T. E., Nucl. Sci. Eng. 121, 286 (1995).

11. Munoz-Cobo, J. L., and Verdu, G., "Noise and Nonlinear Phenomena in Nuclear Systems," Proceedings of a NATO Advanced Research Workshop on Noise and Nonlinear Phenomena in Nuclear Systems, May 23-27, 1988, Valencia, Spain. Edited by J. L. Munoz-Cobo and F. C. Difilippo, p. 59.

12. Difilippo, F. C., Nucl. Sci. Eng., 90, 13-18 (1985).

13. Spencer, R. R., Gwin, R., and Ingle, R., Nucl. Sci. Eng., 80, 603 (1982). 
This page intentionally left blank. 


\section{APPENDIX A}

Start from

$$
\begin{gathered}
\Phi_{A}\left(\omega_{1} \omega_{2}\right)=\left(2_{\pi}\right)^{-2} \int_{-\infty}^{\infty} d \tau_{1} d \tau_{2} \exp \left\{i ( \omega _ { 1 } \tau _ { 1 } + \omega _ { 2 } \tau _ { 2 } ) \int _ { - \infty } ^ { \infty } d t \left(\Phi_{s} \mid \Theta_{1}\left(t-t_{1 f}\right)^{*}\right.\right. \\
\left.* \Theta_{2}\left(t-t_{2 f}\right) \Theta_{3}\left(t-t_{3 f}\right)\right)
\end{gathered}
$$

.express the $\Theta_{q}$-functions in terms of their Fourier transforms:

$$
\Theta_{q}\left(t-t_{q f}\right)=\frac{1}{2 \pi} \int_{-\infty}^{\infty} d \omega_{p} \Theta_{q}\left(\omega_{p}\right) \exp \left(-i \omega_{p}\left(t-t_{q f}\right)\right.
$$

insert now (A.2) into (A.1) to get

$$
\begin{gathered}
\Phi_{A}\left(\omega_{1} \omega_{2}\right)=(2 \pi)^{-5} \overline{v_{p}\left(v_{p}-1\right)\left(v_{p}-2\right)} \int d \tau_{1} d \tau_{2} d t \int d \omega_{3} d \omega_{4} d \omega_{5}^{*} \\
*\left(\Phi_{5} \mid \Theta_{1}\left(\omega_{3}\right) \Theta_{2}\left(\omega_{4}\right) \Theta_{3}\left(\omega_{5}\right)\right) E\left(\omega_{1} \omega_{2}\right)
\end{gathered}
$$

where

$$
\begin{aligned}
E\left(\omega_{1} \omega_{2}\right) & =\exp \left\{i \tau_{1}\left(\omega_{4}+\omega_{1}\right)+i \tau_{2}\left(\omega_{5}+\omega_{2}\right)-i t\left(\omega_{3}+\omega_{4}+\omega_{5}\right)\right. \\
& \left.+i t_{1 f}\left(\omega_{3}+\omega_{4}+\omega_{5}\right)\right\}
\end{aligned}
$$

Next, use the integral representation of the $\delta$-function:

$$
\delta(x)=\frac{1}{2 \pi} \int_{-\infty}^{\infty} d k e^{i k x}
$$

to get

$\Phi_{A}\left(\omega_{1} \omega_{2}\right)=(2 \pi)^{-2} \overline{v_{p}\left(v_{p}-1\right)\left(v_{p}-2\right)} \int d \omega_{3} d \omega_{4} d \omega_{5} \delta\left(\omega_{1}+\omega_{4}\right) \delta\left(\omega_{2}+\omega_{5}\right)$

$$
\delta\left(\omega_{3}+\omega_{4}+\omega_{5}\right)\left(\Phi_{5} \mid \Theta_{1}\left(\omega_{3}\right) \Theta_{2}\left(\omega_{4}\right) \Theta_{3}\left(\omega_{5}\right)\right)
$$

and perform the indicated integrals to get

$\Phi_{A}\left(\omega_{1} \omega_{2}\right)=(2 \pi)^{-2} \overline{v_{p}\left(v_{p}-1\right)\left(v_{p}-2\right)}\left(\Phi_{s} \mid \Theta_{1}\left(\omega_{1}+\omega_{2}\right) \Theta_{2}^{*}\left(\omega_{1}\right) \Theta_{3}^{*}\left(\omega_{2}\right)\right)(\mathrm{A} .7)$ 
Consider next the term

$$
\begin{aligned}
\Phi_{B}\left(\omega_{1} \omega_{2}\right) & =(2 \pi)^{-2} \int_{-\infty}^{\infty} d \tau_{1} d \tau_{2} \exp \left\{i\left(\omega_{1} \tau_{1}+\omega_{2} \tau_{2}\right)\right\} \int_{-\infty}^{\infty} d t\left(\Phi_{5} \mid \Theta_{12} \Theta_{3}\right. \\
+ & \left.\Theta_{13} \Theta_{2}+\Theta_{23} \Theta_{1}\right)
\end{aligned}
$$

where now one expresses the $\Theta_{q q^{\prime}}$ - functions in the form

$$
\Theta_{q q^{\prime}}=\frac{1}{(2 \pi)^{2}} \int d \omega_{3} d \omega_{4} \Theta_{q q^{\prime}}\left(\omega_{3}, \omega_{4}\right) \exp \left\{-i \omega_{3}\left(t-t_{q f}\right)-i \omega_{4}\left(t-t_{q^{\prime} f}\right)\right\}
$$

Again, use of the integral representation of the $\delta$-function yields equaticn $(6.6)$ in the text. 


\section{APPENDIX B}

The Fourier transformed equations (5.15) and (5.16) are

$$
\begin{aligned}
& \left\{\frac{i \omega}{\mathrm{v}}+L_{o}^{+}\right\} p_{q}^{+}(\omega)=D q_{c}(\omega) \\
& \left\{\frac{i}{\mathrm{v}}\left(\omega_{1}+\omega_{2}\right)+L_{o}^{+}\right\} p_{q q^{\prime}}^{+}\left(\omega_{1} \omega_{2}\right)=\overline{v_{p}\left(v_{p}-1\right)} \Sigma_{F} \Theta_{q}\left(\omega_{1}\right) \Theta_{q^{\prime}}\left(\omega_{2}\right)
\end{aligned}
$$

where we used equation (5.18).

Define now the forward Propagator in the frequency domain

$$
\left(\frac{i \omega}{\mathrm{v}}+L o\right) G(1 \mid 2, \omega)=\delta(1-2)
$$

so that the generalized functions, $\mathrm{p}_{\mathrm{q}}$ and $\mathrm{p}_{\mathrm{qq}}$ can be expressed in a closed form as:

$$
\begin{aligned}
& p_{q}^{+}(1, \omega)=\left(D_{q c}(2, \omega) \mid G^{+}(1 \mid 2, \omega)\right) \\
& p_{q q^{\prime}}^{+}\left(1, \omega_{1} \omega_{2}\right)=\overline{v_{p}\left(v_{p}-1\right)}\left(\Sigma_{F}(2) \Theta_{q}\left(2, \omega_{1}\right) \Theta_{q^{\prime}}\left(2, \omega_{2}\right) \mid G^{+}\left(1 \mid 2 \omega_{1} \omega_{2}\right)\right)
\end{aligned}
$$

where we used the reciprocity theorem. $\left(G^{+}(1 \mid 2)=G(2 \mid 1)\right)$

We now proceed to the calculation of the $\Theta_{q}$ and $J_{q}$ in the frequency domain. Fourier transformation of the equations (5.10) and (5.12) yields together with (B.3) the results

$$
\begin{aligned}
& \Theta_{q}(\vec{r}, \omega)=\bar{\gamma}_{q} K_{q}(\omega) h_{R q}(\vec{r}, \omega) \\
& J_{q}(\vec{r}, \omega)=\bar{\gamma}_{q} K_{q}(\omega) h_{s q}(\vec{r}, \omega)
\end{aligned}
$$

with

$$
h_{R q}(\vec{r}, \omega)=\int d v_{1} d \Omega_{1} d v d \Omega \frac{\chi(\mathrm{v})}{4 \pi} \Sigma_{\mathrm{oqc}}\left(\mathrm{v}_{1}\right) G^{+}\left(\vec{r} \mathrm{v} \vec{\Omega} \mid \overrightarrow{\mathrm{r}}_{\mathrm{q}} \mathrm{v}_{1} \vec{\Omega}_{1} \omega\right)
$$




$$
h_{S q}(\vec{r}, \omega)=\int d v_{1} d \Omega_{1} d v d \Omega \frac{\chi_{S(v)}}{4_{\pi}} \Sigma_{\mathrm{oqc}}\left(\mathrm{v}_{1}\right) G^{+}\left(\vec{r} \mathrm{v} \vec{\Omega} \mid \overrightarrow{\mathrm{r}}_{\mathrm{q}} \mathrm{v}_{1} \vec{\Omega}_{1} \omega\right)(\mathrm{B} .8)
$$

The results above are now used for the calculations of the $\Theta_{q q^{\prime}}$ and $J_{q q^{\prime}}$ quantities. We have from the Fourier transform of (5.11) together with (B.4) and (B.5)

$$
\Theta_{q q^{\prime}}\left(\vec{r}_{1} \omega_{1} \omega_{2}\right)=\overline{v_{p}\left(v_{p}-1\right)} \bar{\gamma}_{q} \bar{\gamma}_{q^{\prime}} \mathrm{K}_{q}(\omega) \mathrm{K}_{q^{\prime}}\left(\omega_{2}\right) h_{R q q^{\prime}}\left(\bar{r}, \omega_{1} \omega_{2}\right)
$$

with

$$
\begin{gathered}
h_{R q q^{\prime}}\left(\vec{r} \omega_{1} \omega_{2}\right)=\int d \mathrm{v} d \Omega d \mathrm{v}_{1} d \Omega_{1} \int d \underline{r}_{1}\left[\frac{\chi(\mathrm{v})}{4 \pi} \sum_{F}\left(\mathrm{v}_{1}\right) h_{R q}\left(\vec{r}_{1} \omega_{1}\right) \mathrm{x}\right. \\
\left.h_{R q^{\prime}}\left(\vec{r}_{1} \omega_{2}\right) G^{+}\left(\vec{r} \mathrm{v} \vec{\Omega} \mid 1, \omega_{1} \omega_{2}\right)\right]
\end{gathered}
$$

and

$$
J_{q q^{\prime}}\left(\vec{r}_{1} \omega_{1} \omega_{2}\right)=\overline{v_{p}\left(v_{p}-1\right)} \bar{\gamma}_{q} \bar{\gamma}_{q^{\prime}} \mathrm{K}_{q}\left(\omega_{1}\right) \mathrm{K}_{q^{\prime}}\left(\omega_{2}\right) h_{s q q^{\prime}}\left(\vec{r} \omega_{1} \omega_{2}\right)
$$

where the quantity $h_{s q q^{\prime}}$ is obtained from (B.10) by replacing $\chi(\mathrm{v})$ with $\chi_{s}(\mathrm{v})$. 


\section{APPENDIX C: REVIEW OF THE $\omega$-MODES}

The $\omega$-modes are defined by the transport equation

$$
L_{o o} \Phi_{n^{\prime}}=\frac{\omega n^{\prime}}{\mathrm{V}} \Phi_{n^{\prime}}
$$

where, $\omega_{\mathrm{n}}$, are the time eigenvalues (decay constants associated with the time evolution of the eigenmodes). We also define the adjoint functions

$$
L_{o o}^{+} \Phi_{n}^{+}=\frac{\omega n}{\mathrm{v}} \Phi_{n}^{+}
$$

The pair $\left(\Phi_{n}^{+} ; \Phi_{n}\right)$ satisfy the boundary conditions, bellow, on a convex surface

$$
\Phi_{n}=o ; \text { for } \vec{\Omega} \cdot \overrightarrow{\mathrm{n}}<0 ; \Phi_{\mathrm{h}}^{+}=0 ; \text { for } \vec{\Omega} \cdot \overrightarrow{\mathrm{n}}>0
$$

and the biorthogonality conditions

$$
\left(\Phi_{n^{\prime}}^{+} \mid \frac{1}{\mathrm{v}} \Phi_{n}\right)=N_{o n} \delta_{n n^{\prime}}
$$

The normalization constant, $\mathrm{N}_{\mathrm{on}}$, is given by

$$
N_{o n}=\left(\Phi_{n}^{+} \mid \frac{1}{\mathrm{v}} \Phi_{n}\right)
$$


and has the meaning of an adjoint weighted neutron population.

The eignvalues, $\omega_{n}$, are related to the eigenreactivity, $k_{n}$. To find this relation, form the inner product between, $\Phi_{n}^{+}$, and equation (C.1) and use the form of the $\mathrm{L}_{o 0}$ operator to get

$$
\omega_{n} N_{o n}=\left(D_{n}-P_{n}\right)
$$

$\because$

where the neutron destruction and production terms were defined as

$$
\begin{aligned}
& D_{n}=\left(\Phi_{n}^{+} \mid\left(\Sigma_{T}+\vec{\Omega} \cdot \vec{\nabla}-S_{c}\right) \Phi_{n}\right) \\
& P_{n}=\left(\Phi_{n}^{+} \mid F_{F} \Phi_{n}\right)
\end{aligned}
$$

Divide (C.6) through by, $D_{n}$, to obtain

$$
\left|\omega_{n}\right|=\frac{1}{\Lambda_{n}}\left|\rho_{n}\right|
$$

where we introduced the nth mode reactivity and neutron generation time, $\rho_{\mathrm{n}}$, and $\Lambda_{\mathrm{n}}$, respectively 


$$
\left|\rho_{n}\right|=\left|1-\frac{1}{k_{n}}\right|
$$

$$
\Lambda_{n}=N_{o n} / P_{n}(\mathrm{sec})
$$

and where the nth mode multiplication factor is given by

$$
k_{n}=\frac{P n}{D n}
$$


This page intentionally left blank. 
Nuclear Regulatory Commission

N. Diaz

Polytechnic of Milan

S. A. Pozzi

M. Marsaguerra

University of Tennessee

H. L. Dodds

L. F. Miller

B. R. Upadhyaya

UT-Battelle

L. G. Chiang

F. C. Difilippo

L. Leal

J. K. Mattingly

D. W. McDonald

J. T. Mihalczo

R. B. Oberer

R. B. Perez

V. Protopopescu

T. E. Valentine

J. D. White

M. C. Wright

ORNL Laboratory Records (RC) 
